\title{
Graphs with equal Independence and Annihilation Numbers
}

\author{
C. E. Larson \\ Department of Mathematics and Applied Mathematics \\ Virginia Commonwealth University \\ Richmond VA, USA \\ clarson@vcu.edu \\ R. Pepper \\ Department of Computer and Mathematical Sciences \\ University of Houston-Downtown \\ Houston TX, USA \\ pepperr@uhd.edu
}

Submitted: July 7, 2010; Accepted: Jan 4, 2011; Published: Sep 9, 2011

Mathematics Subject Classification: 05C69

\begin{abstract}
The annihilation number $a$ of a graph is an upper bound of the independence number $\alpha$ of a graph. In this article we characterize graphs with equal independence and annihilation numbers. In particular, we show that $\alpha=a$ if, and only if, either (1) $a \geq \frac{n}{2}$ and $\alpha^{\prime}=a$, or (2) $a<\frac{n}{2}$ and there is a vertex $v \in V(G)$ such that $\alpha^{\prime}(G-v)=a(G)$, where $\alpha^{\prime}$ is the critical independence number of the graph. Furthermore, we show that it can be determined in polynomial time whether $\alpha=a$. Finally we show that a graph where $\alpha=a$ is either König-Egerváry or almost König-Egerváry.
\end{abstract}

\section{Introduction}

An independent set of vertices in a graph is a set of vertices no two of which are adjacent. A maximum independent set is an independent set of largest cardinality. The independence number $\alpha$ is the cardinality of a maximum independent set. Finding a maximum independent set (MIS) in a graph is a well-known widely-studied NP-hard problem [6]. At least partly for this reason, there is a large body of research on finding bounds for the independence number of a graph. The annihilation number is a recent polynomial time computable upper bound for the independence number. It was originally introduced 
in the second author's 2004 dissertation [12], into the wider mathematical literature in $[4,13,3]$, and has led to a new sufficient condition for the existence of a Hamiltonian path in a graph [8].

The annihilation number was originally defined by the second author in terms of a reduction process on the degree sequence (akin to the Havel-Hakimi process; see, for example, $[7,13]$ ). While reading the original proof of Theorem 1.2 (in [12]) Fajtlowicz observed that the largest integer $k$ such that the sum of the smallest $k$ degrees was at most the number of edges, was also an upper bound on the independence number. That this invariant is equivalent to the annihilation number is shown in [12].

Definition 1.1. For a graph $G$ with vertices $V=\left\{v_{1}, v_{2}, \ldots, v_{n}\right\}$, having degrees $d_{i}=$ $d\left(v_{i}\right)$, with $d_{1} \leq d_{2} \leq \ldots \leq d_{n}$, and having $e$ edges, the annihilation number $a=a(G)$ is defined to the the largest index such that $\sum_{i=1}^{a} d_{i} \leq e$.

Theorem 1.2. (Pepper [12]) For any graph $G, \alpha(G) \leq a(G)$.

The second author provided examples in [12] where the annihilation number of a graph is a better upper bound than any of several others, including the minimum of the numbers of non-negative and non-positive eigenvalues (Cvetkovic's bound [2, Thm 3.14]). What follows are two facts that will be used in the characterization of equality. The first is an observation that the annihilation number is at least the floor of half the number of vertices of the graph. The second says that this invariant is monotone with respect to removal of vertices. This is a property it shares with the independence number. For a graph $G$ and a vertex $v \in V(G)$, let $G-v$ be the graph induced on the set $V(G) \backslash\{v\}$. The independence number of a graph $G$ is monotone in the sense that, for any vertex $v \in V(G), \alpha(G-v) \leq \alpha(G)$. A parallel relation holds for the annihilation number.

Observation 1.3. (Pepper [12]) For any graph $G, a(G) \geq\left\lfloor\frac{n(G)}{2}\right\rfloor$.

Lemma 1.4. For a graph $G$ and vertex $v, a(G-v) \leq a(G)$.

Proof. Let $G$ be a graph and $v \in V(G)$. Let $a=a(G)$ and $a^{\prime}=a(G-v)$. It will be shown that $a^{\prime} \leq a$. Let $d_{G}(w)$ be the degree of a vertex $w$ in $G$. For a set $A \subseteq V$ of vertices of $G$, let $d_{G}(A)$ be the sum of the degrees in $G$ of the vertices in $A$. Thus, $d_{G}(A)=\sum_{w \in A} d_{G}(w)$. Let $e=e(G)$ be the size of $G$ and $e^{\prime}=e(G-v)=e(G)-d_{G}(v)$.

Suppose the annihilation number of $G-v$ is at least $a+1$. Then there is a set $A \subseteq V(G-v)$ of $|A|=a+1$ vertices such that $d_{G-v}(A) \leq e^{\prime}$. Then

$$
d_{G}(A) \leq d_{G-v}(A)+d_{G}(v) \leq e^{\prime}+d_{G}(v)=e .
$$

That is, there is a set of $a+1$ vertices in $G$ where the sum of their degrees is less than the number of edges of $G$, and the annihilation number of $G$ is at least $a+1$, contradicting the fact that the annihilation number of $G$ is $a$. So the assumption that $a(G-v)>a(G)$ is false.

In the following, a characterization is given of those graphs with equal independence and annihilation numbers, as well as a polynomial-time algorithm for computing if a graph has this property. 


\section{The Critical Independence Number and the Char- acterization of Equality}

An independent set of vertices $I$ is a critical independent set if $|I|-|N(I)|$ is maximized; these sets were introduced by Zhang [15], who showed that they could be found in polynomial-time. A maximum critical independent set is a critical independent set of maximum cardinality. The critical independence number of a graph $G$, denoted $\alpha^{\prime}=\alpha^{\prime}(G)$, is the cardinality of a maximum critical independent set. The critical independence number of a graph is clearly a lower-bound for the independence number of a graph, and it can be computed in polynomial-time [9].

A graph is independence irreducible if $\alpha^{\prime}=0$. For these graphs the number of neighbors of any independent set of vertices is greater than the number of vertices in the set; fullerene graphs, for instance, are independence irreducible [9]. A graph is independence reducible if $\alpha^{\prime}>0$. A graph is totally independence reducible if $\alpha^{\prime}=\alpha ; K_{2}$ is an example. Determining whether a graph is totally independence reducible can be determined in polynomial-time [9].

Critical independent sets can be extended to maximum independent sets. Butenko and Trukhanov used this discovery to speed up the search for maximum independent sets. This fact is also needed for the proof of Theorem 2.2.

Theorem 2.1. (Butenko and Trukhanov, [1]) If $I_{c}$ is a critical independent set of a graph $G$, then there exists a maximum independent set $I$ of $G$, such that $I_{c} \subseteq I$.

The number of vertices of a graph $G$ is denoted $n=n(G)$. As with $a, \alpha$, and $\alpha^{\prime}$, the name of the graph is usually ommitted when the graph is clear from the context.

Theorem 2.2. (Graphs where $\alpha=a$ ) For a graph $G$, the independence number $\alpha$ equals its annihilation number $a$ if, and only if, either (1) $a \geq \frac{n}{2}$ and $\alpha^{\prime}=a$, or (2) $a<\frac{n}{2}$ and there is a vertex $v \in V(G)$ such that $\alpha^{\prime}(G-v)=a(G)$.

Proof. Let $G$ be a graph with $n$ vertices.

Suppose $a(G) \geq \frac{n(G)}{2}$ and $\alpha^{\prime}(G)=a(G)$. Since $\alpha^{\prime} \leq \alpha \leq a$ for any graph, it follows that $\alpha(G)=a(G)$.

Alternately, suppose that $a(G)<\frac{n(G)}{2}$ and there is a vertex $v$ such that $\alpha^{\prime}(G-v)=$ $a(G)$. Since $\alpha^{\prime}(G-v) \leq \alpha(G-v) \leq \alpha(G) \leq a(G)$ and the first and last terms are equal, every term must be equal and, thus, $\alpha(G)=a(G)$.

Suppose now that $\alpha(G)=a(G)$. It will be shown that either (1) $a(G) \geq \frac{n(G)}{2}$ and $\alpha^{\prime}(G)=a(G)$, or $(2) a(G)<\frac{n(G)}{2}$ and there is a vertex $v \in V(G)$ such that $\alpha^{\prime}(G-v)=$ $a(G)$. The statement can be easily verified for all graphs with three or fewer vertices. Assume that it is true for all graphs with fewer than $n$ vertices.

If $G$ is an empty graph then $\alpha^{\prime}=\alpha=a=n$, and the statement holds. So it can be assumed that $G$ is not empty and, thus, $G$ has a vertex $v$ which is not in every maximum independent set. So $\alpha(G-v)=\alpha(G)=a(G)$. Suppose that $a(G-v)<a(G)$. Then $a(G-v)<\alpha(G-v)$, which contradicts the fact that $\alpha \leq a$ for any graph. Since Lemma 
1.4 implies that $a(G-v) \leq a(G)$, it follows that $a(G-v)=a(G)$ and, furthermore, that $\alpha(G-v)=a(G-v)$. Since $a(G-v)=a(G) \geq\left\lfloor\frac{n(G)}{2}\right\rfloor=\left\lfloor\frac{n(G-v)+1}{2}\right\rfloor$, it follows that $a(G-v) \geq \frac{n(G-v)}{2}$ and thus, by the inductive assumption, that $\alpha^{\prime}(G-v)=a(G-v)$. Then,

$$
\alpha^{\prime}(G-v)=\alpha(G-v)=\alpha(G)=a(G-v)=a(G) .
$$

If $a(G)<\frac{n(G)}{2}$ there is nothing more to show. So assume $a(G) \geq \frac{n(G)}{2}$. In this case it must be shown that $\alpha^{\prime}(G)=a(G)$. Let $J_{c}$ be a maximum critical independent set of $G$. Since it was assumed that $\alpha(G)=a(G), \alpha(G) \geq \frac{n(G)}{2}$ and it follows that $J_{c}$ contains a vertex $u$.

Suppose $N\left(J_{c}\right)=\emptyset$. So $J_{c}$ is an isolated set of vertices and $\alpha^{\prime}\left(G-J_{c}\right)=0$ and $\alpha^{\prime}\left(G-J_{c}+u\right)=1$. Also $\alpha\left(G-J_{c}\right)=\alpha(G)-\left|J_{c}\right|$ and $a\left(G-J_{c}\right)=a(G)-\left|J_{c}\right|$, so $\alpha\left(G-J_{c}\right)=a\left(G-J_{c}\right)$. If $a\left(G-J_{c}\right) \geq \frac{n\left(G-J_{c}\right)}{2}$ then, the inductive assumption implies, $\alpha^{\prime}\left(G-J_{c}\right)=a\left(G-J_{c}\right)$. It then follows that $\alpha\left(G-J_{c}\right)=0$. So $J_{c}$ is also a maximum independent set, $G$ is an empty graph, and $\alpha^{\prime}(G)=\alpha(G)=\left|J_{c}\right|$, proving the statement. If $a\left(G-J_{c}\right)<\frac{n\left(G-J_{c}\right)}{2}$ then $a\left(G-J_{c}\right)=\frac{n\left(G-J_{c}\right)-1}{2}$ and $a\left(G-J_{c}+u\right)=$ $a\left(G-J_{c}\right)+1=\frac{n\left(G-J_{c}\right)-1}{2}+1=\frac{n\left(G-J_{c}\right)+1}{2}=\frac{n\left(G-J_{c}+u\right)}{2}$ and, the inductive assumption implies, $\alpha^{\prime}\left(G-J_{c}+u\right)=a\left(G-J_{c}+u\right)$. It then follows that $\alpha\left(G-J_{c}+u\right)=1$. Since $u$ has no neighbors, this implies that $\alpha\left(G-J_{c}\right)=0$. It again follows that $J_{c}$ is a maximum independent set and $G$ is an empty graph. It was noted earlier that the theorem follows for these graphs.

So it can be assumed that $N\left(J_{c}\right) \neq \emptyset$. Let $w$ be a vertex in $N\left(J_{c}\right)$. It follows from Theorem 2.1 that $J_{c}$ is contained in a maximum independent set $I$. Since $w \notin I, w$ is not in every maximum independent set of $G$. Thus, Equation (1) holds. It is enough to show then that $\alpha^{\prime}(G)=\alpha^{\prime}(G-w)$.

Let $N_{H}(Y)$ be the neighbors of a set $Y$ of vertices in a graph $H$. Since $J_{c}$ is a critical independent set of $G,\left|J_{c}\right|-\left|N_{G}\left(J_{c}\right)\right| \geq|X|-\left|N_{G}(X)\right|$ for any set $X$ of vertices in $G$. Note that $\left|J_{c}\right|-\left|N_{G}\left(J_{c}\right)\right|=\left|J_{c}\right|-\left(\left|N_{G}\left(J_{c}\right)-w\right|+1\right)$. Let $J_{c}^{\prime}$ be a maximum critical independent set in $G^{\prime}=G-w$, so $\alpha^{\prime}(G-w)=\left|J_{c}^{\prime}\right|$. Note that, since $w \in N_{G}\left(J_{c}\right)$, $J_{c} \subseteq V\left(G^{\prime}\right)$. Also, $\left|N_{G^{\prime}}\left(J_{c}\right)\right|=\left|N_{G}\left(J_{c}\right)\right|-1$. Since $J_{c}^{\prime}$ is a critical independent set, $\left|J_{c}^{\prime}\right|-\left|N_{G^{\prime}}\left(J_{c}^{\prime}\right)\right| \geq\left|J_{c}\right|-\left|N_{G^{\prime}}\left(J_{c}\right)\right|=\left|J_{c}\right|-\left(\left|N_{G}\left(J_{c}\right)\right|-1\right)$.

Since $J_{c}^{\prime} \subseteq V\left(G^{\prime}\right), w \notin J_{c}^{\prime}$. There are two cases to consider: (1) the case where $w \in$ $N_{G}\left(J_{c}^{\prime}\right)$, and (2) the case where $w \notin N_{G}\left(J_{c}^{\prime}\right)$. If $w \in N_{G}\left(J_{c}^{\prime}\right)$, then $N_{G}\left(J_{c}^{\prime}\right)=N_{G^{\prime}}\left(J_{c}^{\prime}\right) \cup\{w\}$, and $\left|N_{G}\left(J_{c}^{\prime}\right)\right|=\left|N_{G^{\prime}}\left(J_{c}^{\prime}\right)\right|+1$. Since $\left|J_{c}^{\prime}\right|-\left|N_{G^{\prime}}\left(J_{c}^{\prime}\right)\right| \geq\left|J_{c}\right|-\left|N_{G}\left(J_{c}\right)\right|+1$, it follows that $\left|J_{c}^{\prime}\right|-\left(\left|N_{G}\left(J_{c}^{\prime}\right)\right|-1\right) \geq\left|J_{c}\right|-\left|N_{G}\left(J_{c}\right)\right|+1$ and, thus, $\left|J_{c}^{\prime}\right|-\left|N_{G}\left(J_{c}^{\prime}\right)\right| \geq\left|J_{c}\right|-\left|N_{G}\left(J_{c}\right)\right|$. So $J_{c}^{\prime}$ is a critical independent set of $G$ and $\alpha(G) \geq \alpha^{\prime}(G) \geq\left|J_{c}^{\prime}\right|=\alpha^{\prime}(G-w)=\alpha(G-w)=\alpha(G)$. It follows that $\alpha^{\prime}(G)=\alpha^{\prime}(G-w)$, which was to be shown.

If $w \notin N_{G}\left(J_{c}^{\prime}\right)$, then $N_{G}\left(J_{c}^{\prime}\right)=N_{G^{\prime}}\left(J_{c}^{\prime}\right)$ and $\left|N_{G}\left(J_{c}^{\prime}\right)\right|=\left|N_{G^{\prime}}\left(J_{c}^{\prime}\right)\right|$. So $\left|J_{c}\right|-\left|N_{G}\left(J_{c}^{\prime}\right)\right|=$ $\left|J_{c}^{\prime}\right|-\left|N_{G^{\prime}}\left(J_{c}^{\prime}\right)\right| \geq\left|J_{c}\right|-\left|N_{G^{\prime}}\left(J_{c}\right)\right|=\left|J_{c}\right|-\left(\left|N_{G}\left(J_{c}\right)\right|-1\right) \geq\left|J_{c}\right|-\left|N_{G}\left(J_{c}\right)\right|$. Thus, $J_{c}^{\prime}$ is a critical independent set of $G$ and $\alpha(G) \geq \alpha^{\prime}(G) \geq\left|J_{c}^{\prime}\right|=\alpha^{\prime}(G-w)=\alpha(G-w)=\alpha(G)$, proving in this case too that $\alpha^{\prime}(G)=\alpha^{\prime}(G-w)$.

Let $G$ be a graph. The steps to determine whether $\alpha(G)=a(G)$ are as follows. 

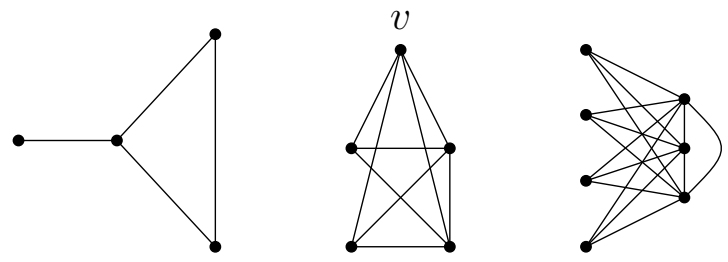

Figure 1: Graphs where $\alpha=a$. For the graphs on the left and center $\alpha=a=2$. For the graph on the right, $\alpha=a=4$. The graphs on the left and right are Type I graphs. These graphs are also examples of non-bipartite König-Egerváry (KE) graphs. The graph in the center is a Type II graph. This graph is almost KE. If the vertex $v$ is removed the graph induced on the remaining vertices is $\mathrm{KE}$ and has a perfect matching.

1. Calculate $a(G)$.

2. If $a(G) \geq \frac{n}{2}$, calculate $\alpha^{\prime}(G)$. If $\alpha^{\prime}(G)=a(G)$, then Theorem 2.2 implies that $\alpha(G)=a(G)$. If $\alpha^{\prime}(G) \neq a(G)$, then Theorem 2.2 implies that $\alpha(G) \neq a(G)$.

3. If $a(G)<\frac{n}{2}$, choose an edge $v w$. It cannot be that both vertex $v$ and vertex $w$ are in every maximum independent set of $G$. Calculate $\alpha^{\prime}(G-v)$ and $\alpha^{\prime}(G-w)$. If $a(G)=\alpha^{\prime}(G-v)$ or $a(G)=\alpha^{\prime}(G-w)$ then Theorem 2.2 implies that $\alpha(G)=a(G)$.

The proof of Theorem 2.2 actually shows that if $\alpha(G)=a(G), a(G)<\frac{n(G)}{2}$, and vertex $u$ is not in every maximum independent set of $G$, then $\alpha^{\prime}(G-u)=a(G)$. Since either $v$ or $w$ is not in every maximum independent set of $G$ then, if $a(G) \neq \alpha^{\prime}(G-v)$ and $a(G) \neq \alpha^{\prime}(G-w)$, it follows that $\alpha(G) \neq a(G)$.

Since $a$ and $\alpha^{\prime}$ can be calculated in polynomial time, and since the preceding algorithm will terminate after at most three calculations of these invariants, it can be determined in polynomial time whether $a=\alpha$.

\section{Graphs where $\alpha=a$ are (almost) König-Egerváry}

Observation 1.3 says that, for any graph, either $a \geq \frac{n}{2}$ or $a=\frac{n-1}{2}$. In the former case, Theorem 2.2 says that graphs $G$ where $\alpha(G)=a(G)$ have the property that $\alpha^{\prime}(G)=$ $\alpha(G)=a(G)$ (call these graphs Type I graphs). In the latter case, the theorem implies that that graphs $G$ where $\alpha(G)=a(G)$ have the property that there is a vertex $v \in V(G)$ such that $\alpha^{\prime}(G-v)=\alpha(G-v)=a(G-v)=a(G)=\alpha(G)$ (call these Type II graphs).

A matching in a graph is a set of pairwise non-incident edges. A graph has a perfect matching if there is a matching which saturates all of the vertices of the graph (that is, every vertex is incident to one of the edges of the matching). The matching number $\mu$ of a graph is the cardinality of a largest matching in the graph. If the graph has a perfect matching, then the number of vertices must be even and $\mu=\frac{n}{2}$. The König-Egerváry Theorem says that, for bipartite graphs, $\alpha+\mu=n$ [11]. This relation holds true for some 
non-bipartite graphs as well: the left-most graph in Figure 1 is an example. The class of graphs for which the relation $\alpha+\mu=n$ holds are called König-Egerváry graphs (or, more succinctly, KE graphs). These graphs were first characterized by Deming [5] and Sterboul [14] in 1979, and new characterizations and research have been ongoing. We call a graph $G$ almost König-Egerváry (or almost $K E$ ) if $G$ is not $\mathrm{KE}$, but for which there is a vertex $v \in V(G)$ such that $G-v$ is $\mathrm{KE}$ (the middle graph in Figure 3 is an example).

The relation between KE graphs and the graphs described in Theorem 2.2 comes from the following result:

Theorem 3.1. (Larson, [10]) For a graph $G, \alpha^{\prime}(G)=\alpha(G)$ if, and only if, $G$ is a KE graph.

Graphs of Type I are KE graphs; and graphs of Type II almost are - these have a vertex $v$ which, when removed, yields a KE graph.

The characterization of the graphs in Theorem 2.2 is somewhat formal and unintuitive. Our goal in this section is to give a more intuitive picture of the graphs which have equal independence and annihilation numbers. In the case where these invariants are equal and $a \geq \frac{n}{2}$, the graphs are a subclass of the KE graphs. In the other case, where $\alpha=a$ and $a=\frac{n-1}{2}$, the graphs are a subclass of the almost KE graphs. The following two theorems flesh out this picture.

For a graph $G$ and a set $S \subseteq V(G)$, let $S^{c}=V(G) \backslash S$. Note that if $I$ is a maximum independent set, then $I^{c}=N(I)$. For $S \subset V(G)$, let $d(S)=d_{G}(S)=\sum_{v \in V(G)} d(v)$. An annihilating set $A$ is a set whose degree sum $d(A)$ is no more than the number of edges $e$ of the graph. The name is chosen for the reason that a set of vertices corresponding to the lowest $a$ degrees in a graph has this property. An annihilating set $A$ is maximal if, for every vertex $v \in A^{c}, d(A \cup\{v\})>e$; it is maximum if $|A|=a$.

Lemma 3.2. (The Matching Lemma, L., [10]) If $I_{c}$ is a critical independent set, then there is a matching from $N\left(I_{c}\right)$ into $I_{c}$.

Theorem 3.3. (Type I graphs) For a graph $G$ with $a(G) \geq \frac{n(G)}{2}, \alpha(G)=a(G)$ if, and only if, $G$ is a $K E$ graph and every maximum independent set of $G$ is a maximum annihilating set.

Proof. Let $G$ be a graph with $a(G) \geq \frac{n(G)}{2}$.

Suppose that $\alpha(G)=a(G)$. So $G$ is a Type I graph and $\alpha^{\prime}(G)=\alpha(G)=a(G)$. Let $I_{c}$ be a maximum critical independent set. Since $\alpha^{\prime}(G)=\left|I_{c}\right|, \alpha(G)=\left|I_{c}\right|$, and $I_{c}$ is a maximum independent set. The Matching Lemma implies that there is a matching from $N\left(I_{c}\right)$ into $I_{c}$. So $\mu(G) \geq\left|N\left(I_{c}\right)\right|$. Then $\alpha(G)+\mu(G) \geq\left|I_{c}\right|+\left|N\left(I_{c}\right)\right|=n(G)$. Since for any graph $\alpha+\mu \leq n$, we have $\alpha(G)+\mu(G)=n(G)$, and $G$ is a KE graph. Let $I$ be any maximum independent set of $G$. Since $a(G)=\alpha(G)=|I|, I$ is a maximum annihilating set.

Suppose now that $G$ is a KE graph and every maximum independent set of $G$ is a maximum annihilating set. Let $I$ be a maximum independent set. By definition, $\alpha(G)=|I|$ and $a(G)=|I|$. So $\alpha(G)=a(G)$. 


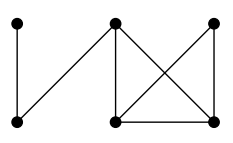

Figure 2: A KE graph where $\alpha \neq a$. Here $\alpha=3$ and $a=4$.

There are polynomial time algorithms both for recognizing KE graphs and for finding maximum independent sets in KE graphs: one justification of these facts is via Theorem 3.1 and the existence of a polynomial time algorithm for finding the critical independence number $\alpha^{\prime}[9]$. Note too that there are KE graphs where $\alpha \neq a$ (see Figure 2). Theorem 3.3 says that Type I graphs are KE, which helps in understanding these graphs, but the second part of that characterization is less helpful. Suppose a graph is KE. Is the graph of Type I? The only way to check is to calculate the critical independence and annihilation numbers directly and compare. It seems, though, that something more intuitive might be true, namely, that given a KE graph $G$ and maximum independent set $I$, if $I^{\prime}$ is formed by adding any vertex in $I^{c}$ to $I$, then $I^{\prime}$ is no longer an annihilating set, that is, that $I$ is a maximal annihilating set.

Conjecture 3.4. For a graph $G$ with $a \geq \frac{a}{2}, \alpha=a$ if, and only if, $G$ is $K E$ and every maximum independent set of $G$ is a maximal annihilating set.

We now turn to a discussion of Type II graphs.

Lemma 3.5. A graph $G$ is an almost $K E$ graph if, and only if, there is a vertex $v \in V(G)$ such that $G-v$ is a KE graph, $\alpha(G-v)=\alpha(G)$, and $\mu(G-v)=\mu(G)$.

Proof. Suppose $G$ is an almost KE graph. By definition, there is a vertex $v \in V(G)$, such that $G-v$ is a KE graph. That is, $\alpha(G-v)+\mu(G-v)=n(G-v)=n(G)-1$. Now $\alpha(G) \geq \alpha(G-v)$, and $\mu(G) \geq \mu(G-v)$. Suppose $\alpha(G)>\alpha(G-v)$. Then $\alpha(G)=\alpha(G-v)+1$, and $\alpha(G)+\mu(G) \geq[\alpha(G-v)+1]+\mu(G-v)=n(G-v)+1=n(G)$. So $G$ is a KE graph, contradicting our assumption. So $\alpha(G)=\alpha(G-v)$. Similarly, if $\mu(G)>\mu(G-v)$ a contradiction ensues. Thus $\mu(G)=\mu(G-v)$, which was to be proved.

Now suppose that $G$ is a graph with a vertex $v \in V(G)$ such that $G-v$ is a KE graph, $\alpha(G-v)=\alpha(G)$, and $\mu(G-v)=\mu(G)$. It must be shown that $G$ is not a KE graph. Since $G-v$ is a KE graph, we have $\alpha(G-v)+\mu(G-v)=n(G-v)=n(G)-1$. So $\alpha(G)+\mu(G)=n(G)-1$, which proves the claim.

Theorem 3.6. (Type II graphs) If $G$ is a graph where $a(G)=\frac{n(G)-1}{2}$, then $\alpha(G)=a(G)$ if, and only if, $G$ is almost $K E$ and, for any vertex $v \in V(G)$ such that $G-v$ is $K E$, $G-v$ has a perfect matching.

Proof. Let $G$ be a graph where $a(G)=\frac{n(G)-1}{2}$.

Suppose first that $\alpha(G)=a(G)$. If $G$ were KE then $\mu(G)=\frac{n(G)+1}{2}$, which is impossible; so $G$ is not KE. Theorem 2.2 implies that there is a vertex $w \in V(G)$ such that $\alpha^{\prime}(G-w)=\alpha(G-w)=a(G-w)=a(G)=\alpha(G)$. Since $\alpha^{\prime}(G-w)=\alpha(G-w)$, 
Theorem 3.1 implies that $G-w$ is KE. So $G$ is almost KE. Now consider any vertex $v \in V(G)$ such that $G-v$ is KE. By definition, $\alpha(G-v)+\mu(G-v)=n(G-v)$. This fact, together with the fact that $G$ is not KE, implies that $\alpha(G)=\alpha(G-v)$. Then $\alpha(G-v)=a(G)=\frac{n(G)-1}{2}=\frac{n(G-v)}{2}$, and it follows that $\mu(G-v)=\frac{n(G-v)}{2}$, which means that $G-v$ has a perfect matching.

Now suppose that $G$ is almost KE and, for any vertex $v \in V(G)$ such that $G-v$ is $\mathrm{KE}, G-v$ has a perfect matching. Since $G$ is almost KE, Lemma 3.5 implies that there is a vertex $v \in V(G)$ such that $G-v$ is a KE graph, $\alpha(G-v)=\alpha(G)$, and $\mu(G-v)=\mu(G)$. By definition, $\alpha(G-v)+\mu(G-v)=n(G-v)$. Since $G-v$ has a perfect matching, it follows that $\mu(G-v)=\frac{n(G-v)}{2}$. So $\alpha(G-v)=\frac{n(G-v)}{2}$. Since $\alpha(G-v) \leq \alpha(G) \leq a(G)$, and the first and last terms are equal, it follows that $\alpha(G)=a(G)$, which was to be proved.

The authors would like to thank one of the referees who wrote particularly detailed comments that led to significant improvement in the clarity of our presentation.

\section{References}

[1] S. Butenko and S. Trukhanov, Using Critical Sets to Solve the Maximum Independent Set Problem, Operations Research Letters 35(4) (2007) 519-524.

[2] D. M. Cvetkovic, M. Doob, H. Sachs, Spectra of Graphs, 3rd revised ed., Johann Ambrosius Barth Verlag, Heidelberg, 1995.

[3] E. DeLaVina, C. E. Larson, R. Pepper and B. Waller, Grafitti.pc on the 2-domination number of a Graph, Congressus Numerantium 203, 2010, 15-32.

[4] E. DeLaVina, R. Pepper and B. Waller, Independence, radius and Hamiltonian paths, MATCH Commun. Math. Comput. Chem: proceedings of the conference "Computers in Scientific Discovery III", Ghent, February 6-9, 2006 (eds. G. Brinkmann, P. W. Fowler) (2007) Vol. 58, pp. 481-510.

[5] R. W. Deming, Independence Numbers of Graphs - an Extension of the KoenigEgervary Theorem, Discrete Mathematics, 27 (1979) 23-33.

[6] M. Garey and D. Johnson, Computers and Intractability, W. H. Freeman and Company, New York, 1979.

[7] J. Griggs and D. Kleitman, Independence and the Havel-Hakimi Residue, Discrete Mathematics 127 1-3 (1994) 209-212.

[8] L. Jennings, New Sufficient Condition for Hamiltonian Paths, dissertation, Rice University, 2008.

[9] C. E. Larson, A Note on Critical Independent Sets, Bulletin of the Institute of Combinatorics and its Applications, 51 (2007), 34-46.

[10] C. E. Larson, The Critical Independence Number and an Independence Decomposition, European Journal of Combinatorics 32(2), 2011, 294-300. 
[11] L. Lovasz, M. D. Plummer, Matching Theory, North Holland, Amsterdam, 1986.

[12] R. Pepper, Binding Independence, Ph.D. Dissertation, University of Houston, 2004.

[13] R. Pepper, On the Annihilation Number of a Graph, Proceedings of the 15th American Conference on Applied Mathematics, World Scientific and Engineering Academy and Society, 2009, 217-220.

[14] F. Sterboul, A characterization of the graphs in which the transversal number equals the matching number, Journal of Combinatorial Theory. Series B, 1979 vol:27, 228229.

[15] C.-Q. Zhang, Finding critical independent sets and critical vertex subsets are polynomial problems, SIAM J. Discrete Mathematics, 3:431-438, 1990. 